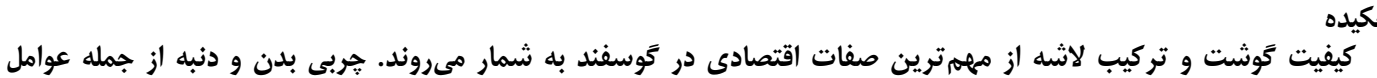

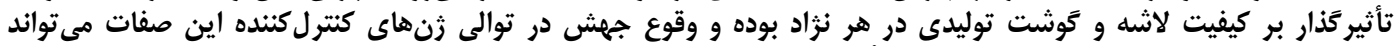

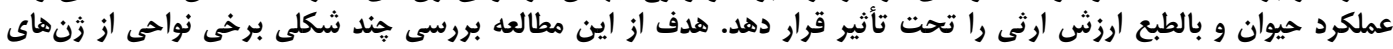

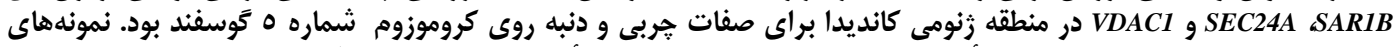

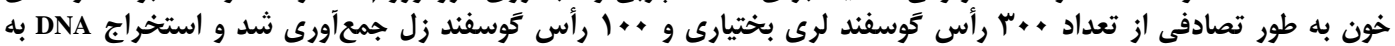

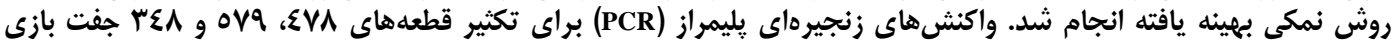

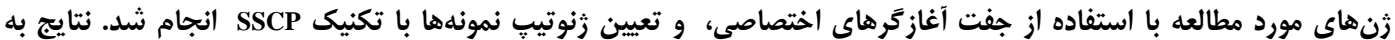

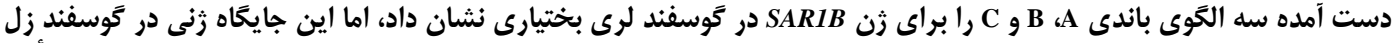

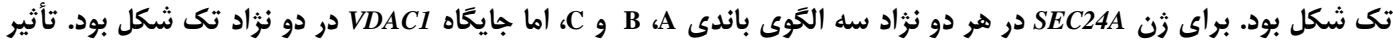

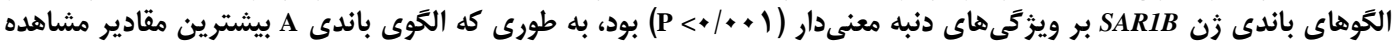

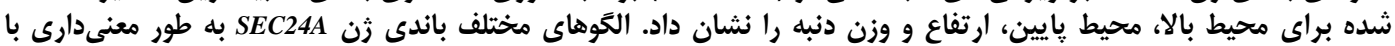

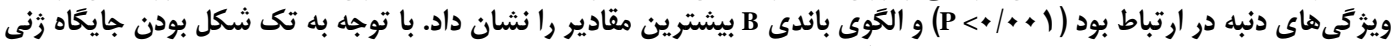

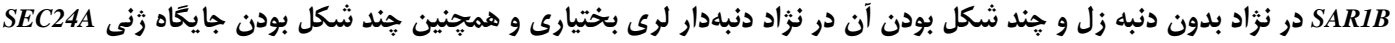

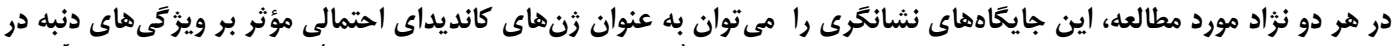

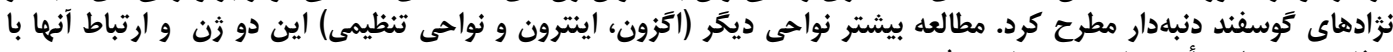

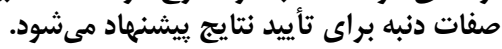

وازههاى كليدى: تَوسفند، صفات دنبه، SDAC1، SEC24A، SAR1B

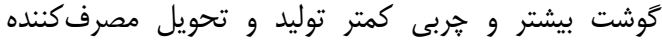

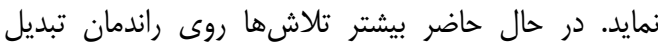

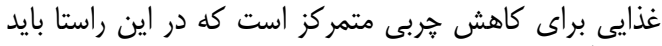

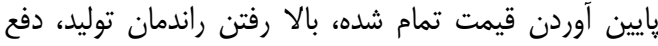

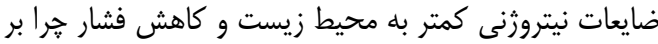

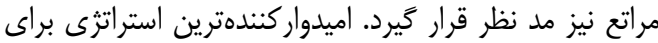

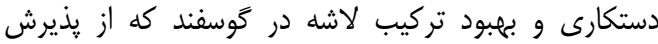

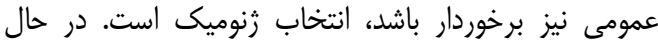

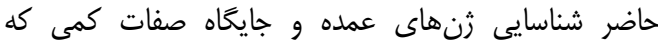

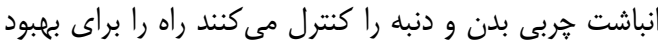

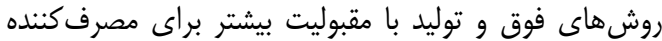

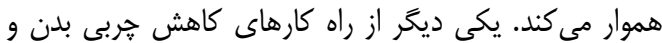

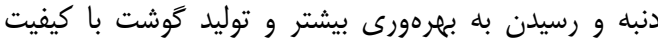

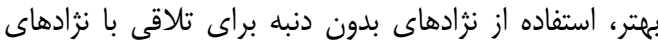

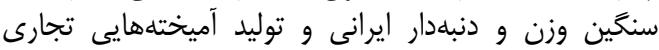

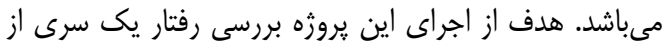

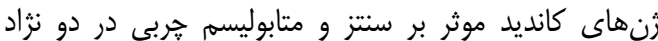
كوسفند لرى بختيارى و زل بوده ئر است.

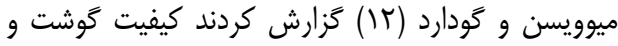

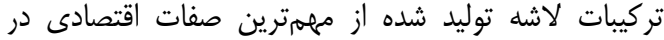

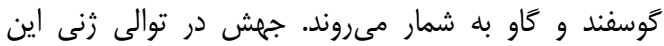

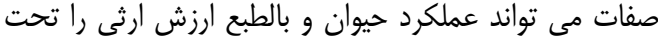

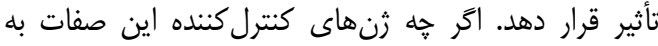

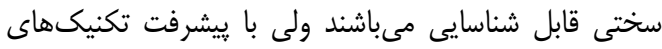

داشتن دنبه از ويزگىهاى اكثر گوسفندان از جمله

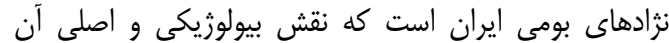

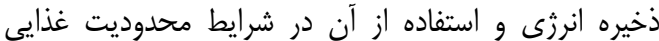

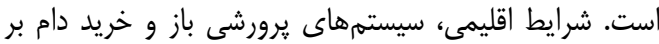

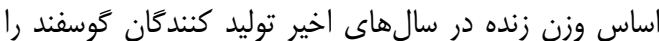

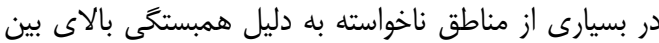

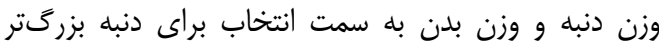

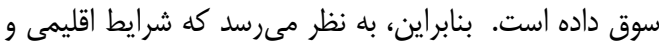

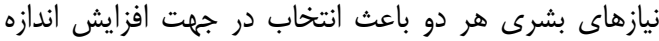

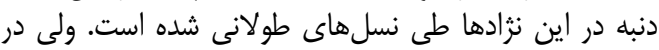

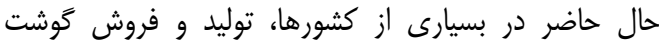

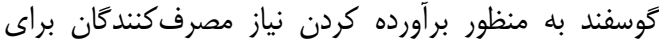

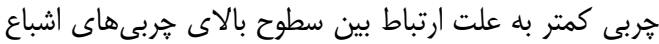

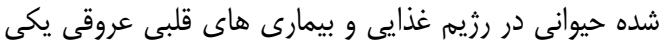

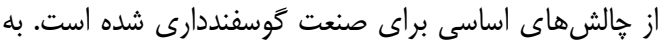

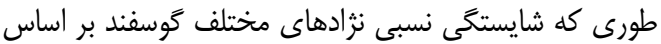

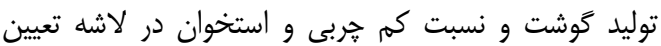

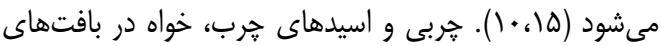

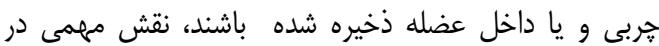

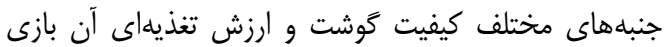
مى كنند.

مصرف كنندگان لاشه با هربى كمتر را مى يسندند. بنابراين

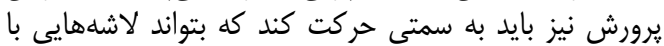


حدود •ب هزار نو كلئوتيد مىباشد (1). يكى از بروتئينهاي

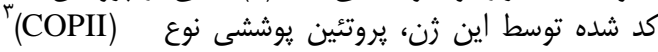

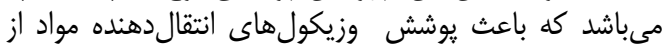

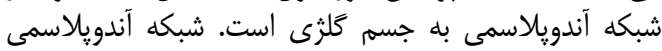

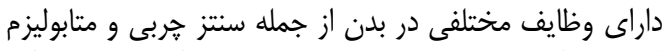

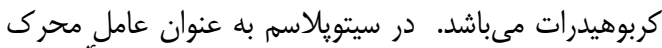

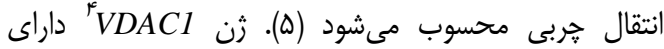

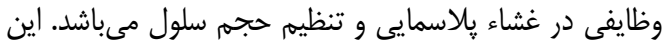

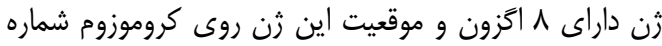

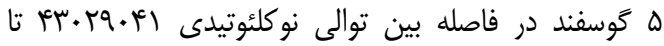

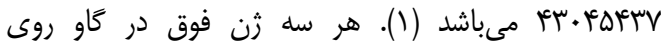

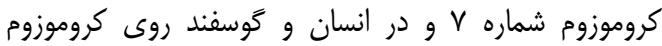

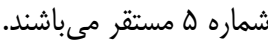

با توجه به اينكه تاكنون تحقيقى در رابطه با با ارتباط اين

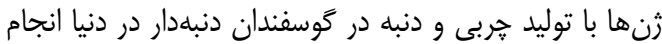

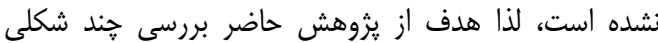

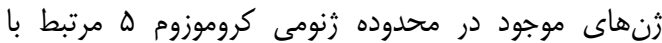

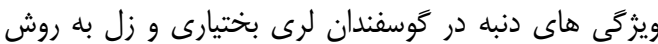
PCR-SSCP

مواد و روشها

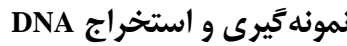

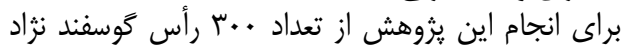

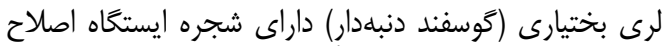

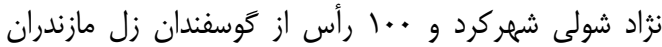

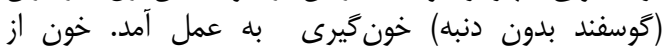

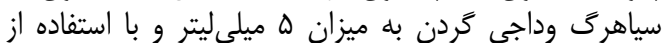

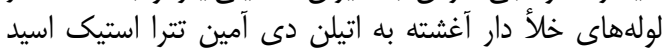

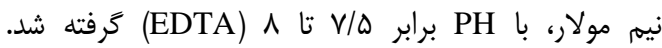

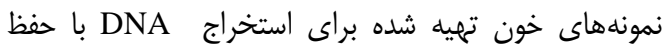

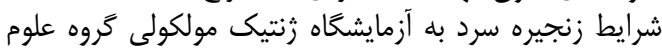

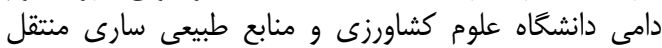

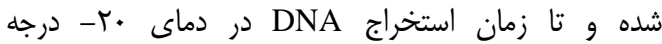

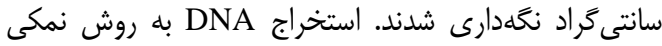

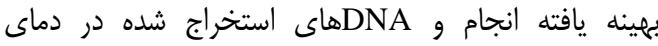

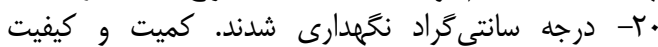

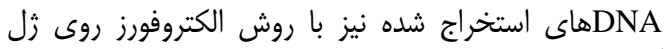

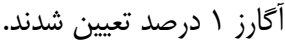

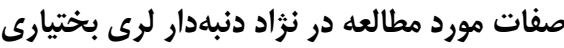

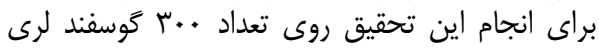

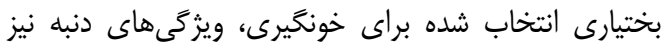

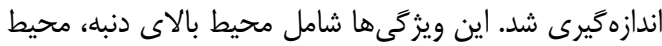

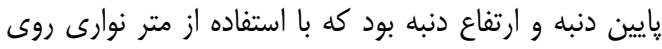

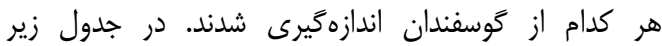

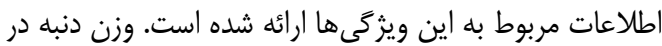

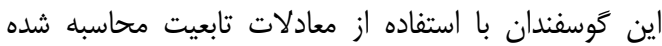
براى اين كوسفند (·r) برآورد كرديد.

1- Secretion Associated, Ras Related GTPase 1B 3- Coat Protein Complex II

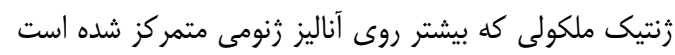

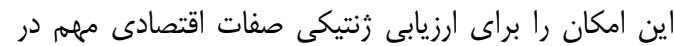

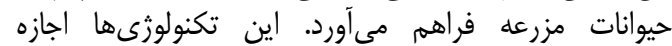

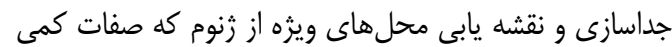

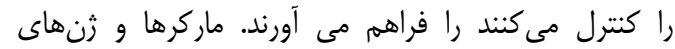

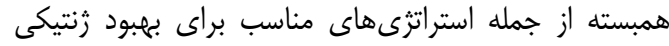
اين صفات مى باشند (سآس).

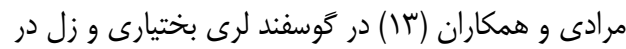

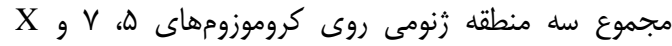

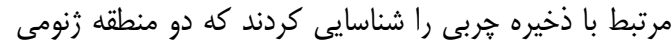

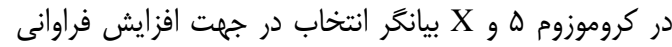

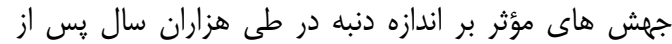

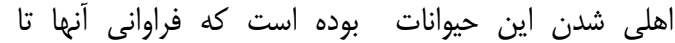

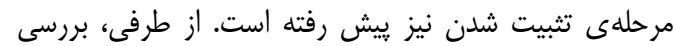

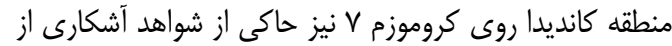

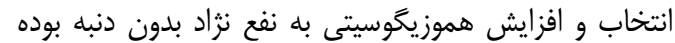

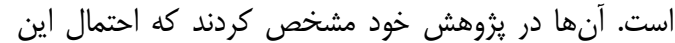

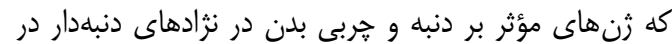

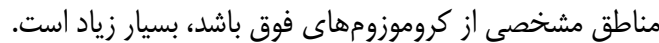

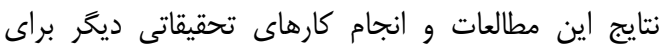

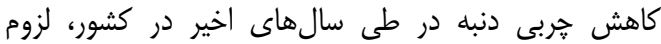

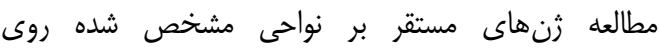

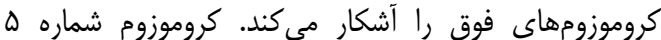
داراى سه SNP با نشانههاى انتخاب مثبت و و معنى كردار به نامهاى

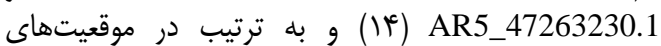
دار

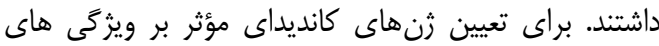
دنبه محدوده r مخابازى اطراف اين SNP ها مورد كاوش قران هاري

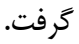

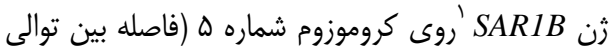

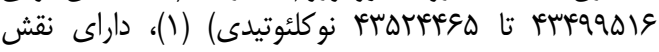

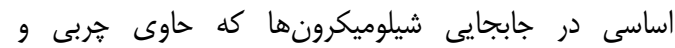

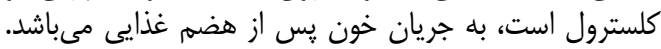

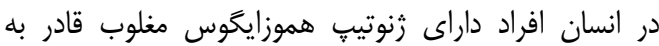

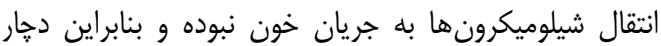

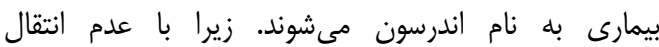

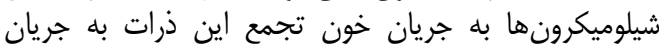

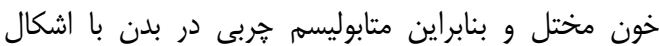

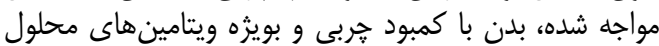

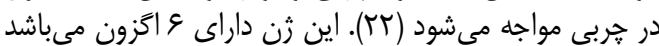

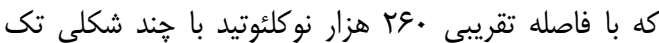

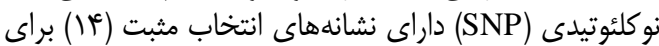

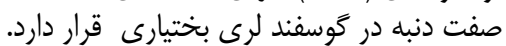

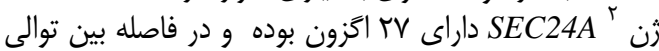
شاره

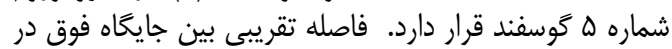

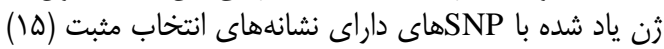
2-SEC24 familv member A 4- Voltage-Dependent Anion Channel 
جدول ا- آماره هاى توصيفى ابعاد دنبه و وزن آن در گوسفند لرى بختيارى Table 1. Descriptive statistics of fat tail dimensions and weights in Lori-Bakhtiari sheep breed

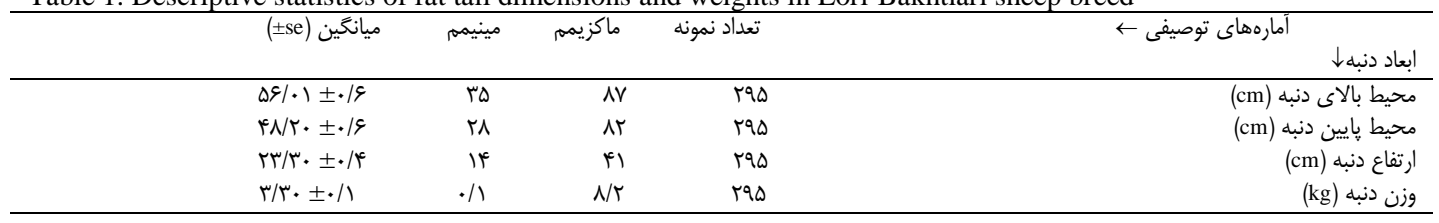

اختماصى با انتخاب شدند. آغازكرهاى SAR1B, VDAC1, SEC24A

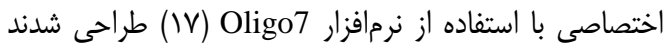

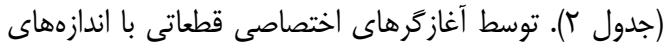

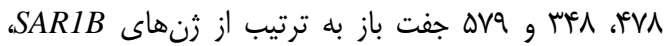

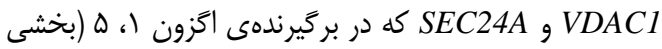

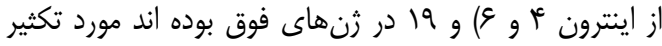

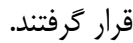

\section{تكثير قطعه مورد نظر با استفاده از واكنث زنجيرهاى}

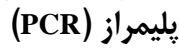

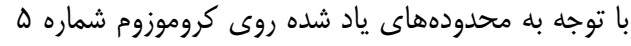

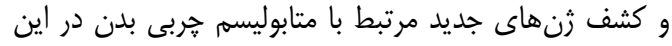

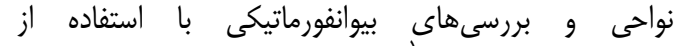

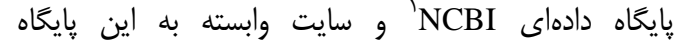
(GeneCards)

جدول r- آغازكَرها و شرايط اختصاصى هر كدام از آنها براى تكثير قطعات DNA Table 2. The primers and their special conditions for DNA amplification

\begin{tabular}{|c|c|c|c|c|}
\hline طول قطعه (bp) & GC & دما & توالى ('........3. & 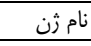 \\
\hline \multirow{2}{*}{ Fra } & $\Delta \Delta / \cdot$ & $\Delta \Lambda / \Gamma$ & F- GTGTCCTTTCCAGATGGTCG & \multirow{2}{*}{ SARIB } \\
\hline & $\Delta \Delta / \cdot$ & $\Delta V / F$ & R- TCCCCAAGCTAAGAACCG & \\
\hline \multirow{2}{*}{ MFA } & $\Delta \cdot /$. & $\Delta \% /$. & F- ACGTTAGTGTGAAGCTGG & \multirow{2}{*}{ VDACl } \\
\hline & $e V / s$ & $\Delta F /$. & R- TGACTTTGCCTTCCTACTC & \\
\hline \multirow{2}{*}{ avq } & $\Delta \Delta /$. & $\Delta \omega / V$ & F- GGTAGTCTTCTTCTCTGAACG & \multirow{2}{*}{$S E C 24 A$} \\
\hline & $\Delta \Delta /$. & $\Delta \Delta /$. & R- CATTTAGAACCTGCAAGCTG & \\
\hline
\end{tabular}

مخلوط شد و سبّ در دستخاه ترموسايكلر به مدت • ماد دقيقه

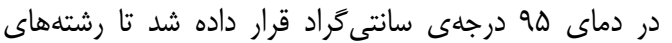

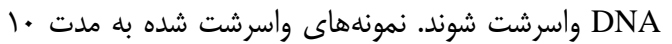

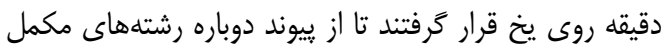

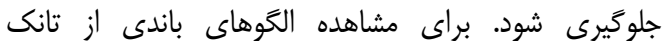

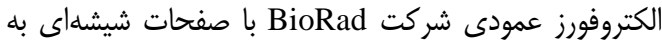

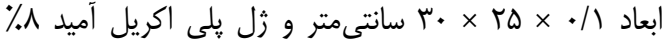

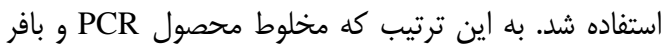

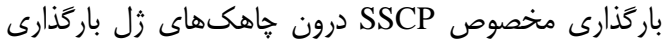

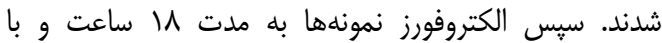

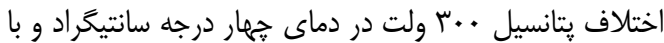

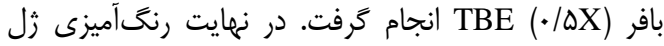

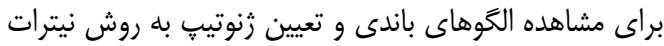
نقره انجام شد.
شرايط دمائى و زمانى PCR در جدول ب ج نشان داده شد.

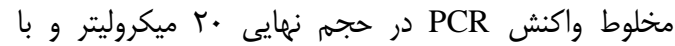

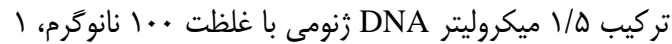

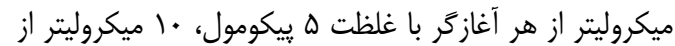

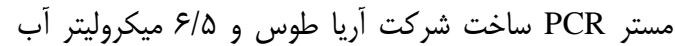

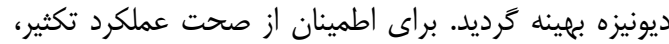
محصولات PCR روى زل آَارز يك درصد الكتروفورز شدند

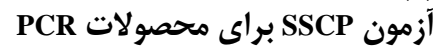

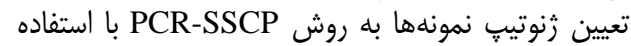

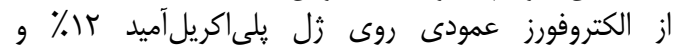

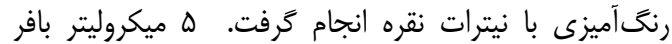

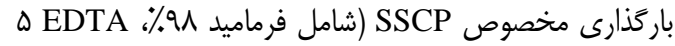
PCR مولار، برموفنل بلو •(\%) با هـ ميكروليتر محصول

جدول س- دما و زمان در هر يك از مراحل واكنش زنجيرهاى يلىمراز (PCR) براى ثنهاى مختلف Table 3. Temperature and time in each step of PCR for different genes

\begin{tabular}{|c|c|c|c|c|c|c|}
\hline بسط يكنواخت (ه) & $\begin{array}{l}\text { تكثير } \\
\text { (๘) }\end{array}$ & 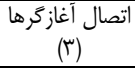 & واسرشته سازى & واسرشته سازى اوليه & شرايط مراحل & نام ثن \\
\hline VT & VT & gr & 90 & 90 & $\left(\mathrm{o}^{\mathrm{C}}\right)$ los & \multirow{2}{*}{ SARIB } \\
\hline Fr. & is & in & is & r.. & زمان (ثانيه) & \\
\hline vr & vr & Df & 90 & 90 & $\left(\mathrm{o}^{\mathrm{C}}\right)$ (م) & \multirow{2}{*}{$V D A C l$} \\
\hline Fr. & id & $i \Delta$ & $i \Delta$ & r.. & زمان (ثانيه) & \\
\hline$v^{2}$ & vr & $\Delta V$ & 90 & 90 & $\left(\mathrm{o}^{\mathrm{C}}\right)^{\mathrm{s}}$ & \multirow{2}{*}{$S E C 24 A$} \\
\hline Fr. & is & iQ & iQ & r.. & زمان (ثانيه) & \\
\hline
\end{tabular}

1- National Centre for biotechnology Information 
در دو نزاد با استفاده از تست دقيق فيشر و با كمك نسخه

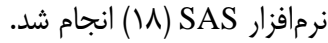

\section{نتايج و بحث}

قطعه مربوط به اخزون شماره ها، بخشى از ايتترون أ و و

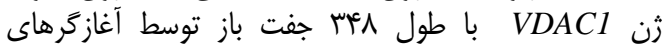
اختصاصى تكثير شد. قطعه تكثير شده فوق بار با استفاده از روش

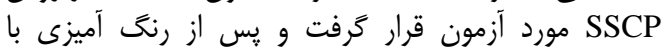

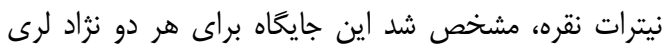

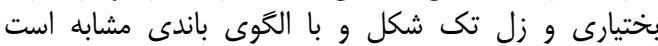

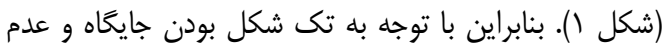

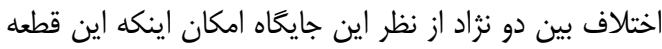

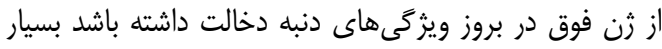

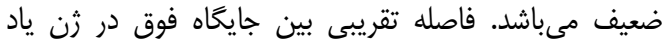

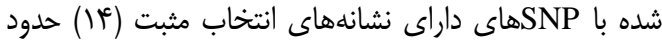
• 11 .

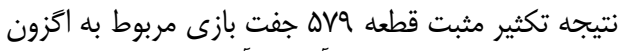

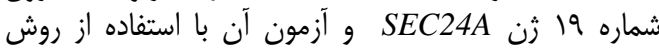

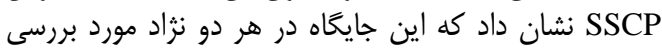

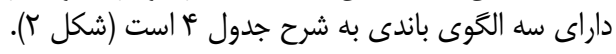

تجزيه و تحليل آمارى

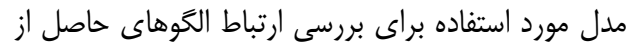

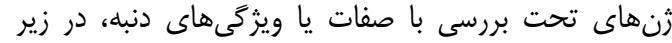

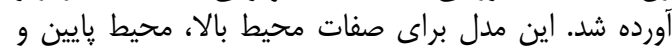

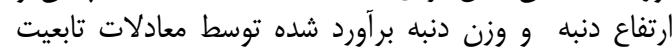

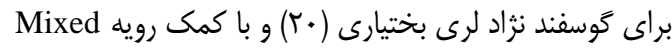

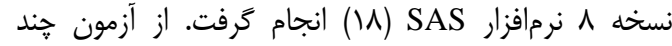

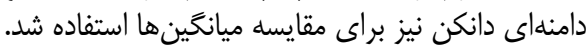

(1) دابطه دابنه

$\mathrm{Y}_{\mathrm{ijkl}}=\mu+\mathrm{R}_{\mathrm{i}}+\mathrm{G}_{\mathrm{j}}+\mathrm{S}_{\mathrm{k}}+\mathrm{A}_{\mathrm{l}}+\mathrm{e}_{\mathrm{ijkl}}$

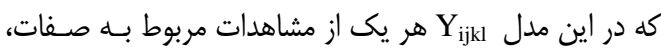

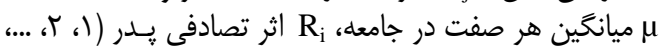

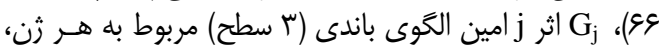

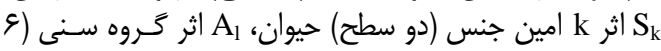

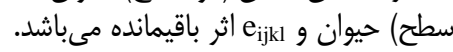

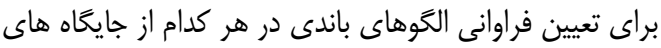
زنى مورد نظر از نرمافزار استفاده شد. مقايسه بين فراوانى الخوهاى باندى مشاهده شده

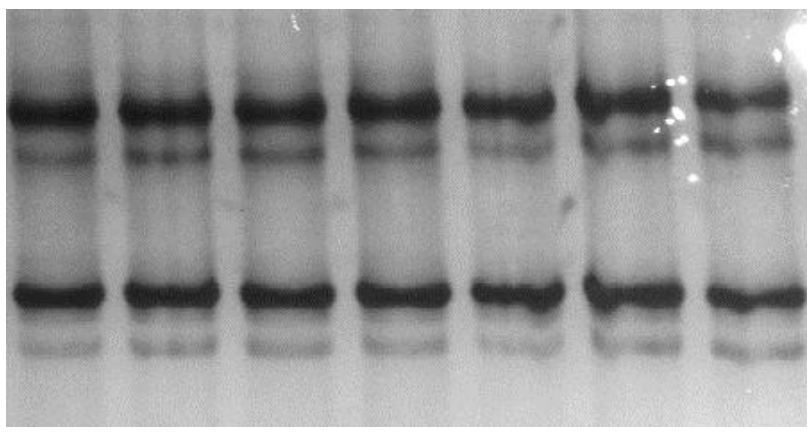

شكل ا- الخوى باندى مشاهده شده براى زن VDAC1 در دو نزاد زل و لرى بختيارى (هر دو نزاد يك شكل بودند) Figure 1. SSCP banding pattern for VDACl gene in Zel and Lori-Bakhtiari sheep breeds (Both breeds were monomorph)

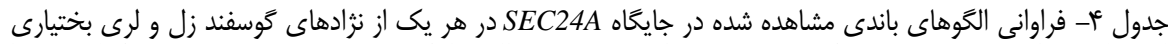
Table 4. The number and frequency (percentage) of banding patterns in exon 19 of SEC24A gene in Zel and

\begin{tabular}{|c|c|c|c|c|}
\hline \multicolumn{2}{|c|}{$\mathrm{C}$} & B & A & الكوهاى باندى + \\
\hline درصد & فراوانى & فراوانى درصد & فراوانى درصد & نزاد ${ }^{2}$ \\
\hline$f$. & $r$. & rr & rA $\quad$ M & زل \\
\hline r & 9. & $11 \mathrm{~V}$ & $11 \pi$ & لرى بختيارى \\
\hline ro & $q$. & 141 & אזו & مجموع \\
\hline
\end{tabular}

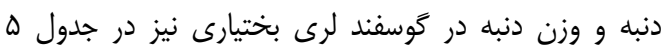

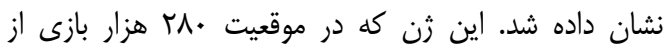

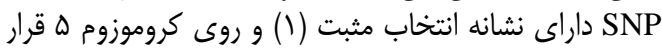

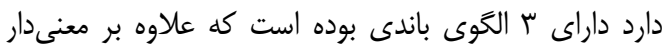

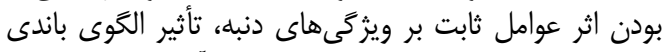

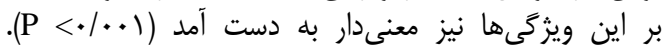

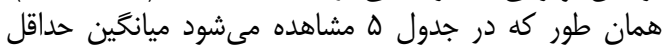

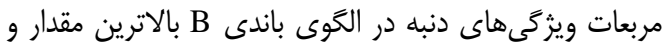

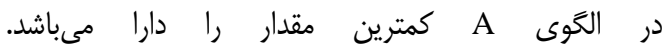

با توجه به نتايج به دست آمده در مجموع فراوانى الكَوهاى

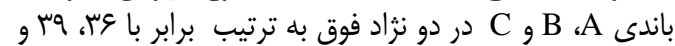

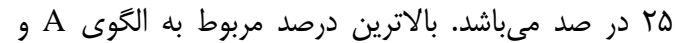

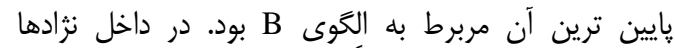

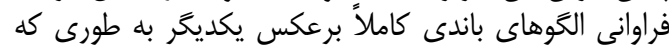

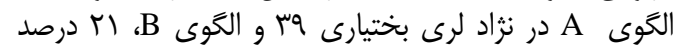

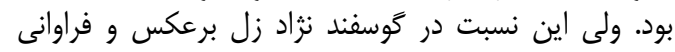

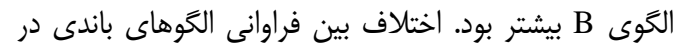

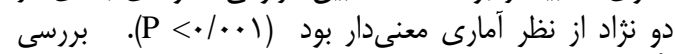

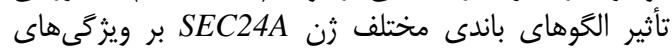




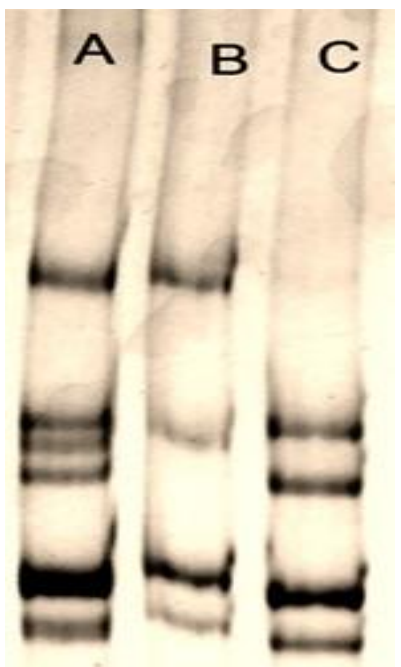

شكل r- الكوهاى باندى مشاهده شده براى ثن SEC24A در دو نثاد زل و لرى بختيارى

Figure 2. SSCP banding patterns for SEC24A gene in Zel and Lori-Bakhtiari sheep breeds

ديخر بيمارىهاى مرتبط با بالا بودن سطح كلسترول ييشنهاد

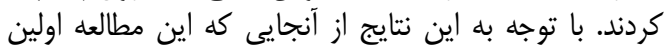

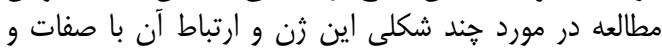

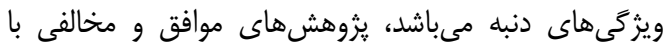

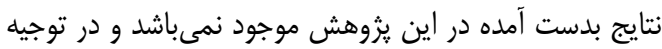

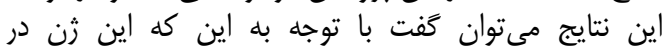

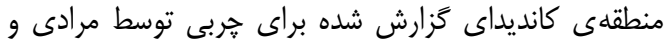

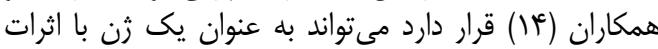

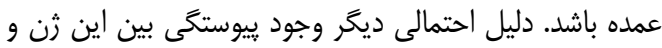

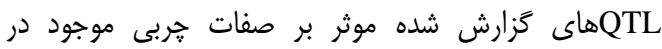

نزديكى اين ثن مى باشد (1) (I).
تحقيقاتى كه روى موش و انسان صورت كر فتنه نشان ميدهد هر كدام از خانوادههاى زئ SEC

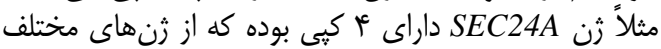

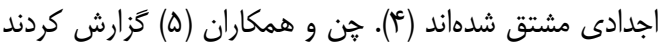

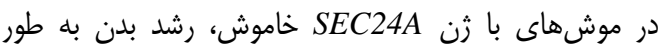

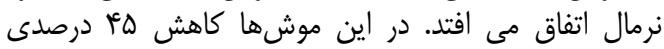

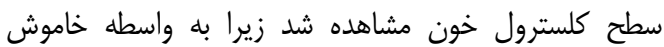

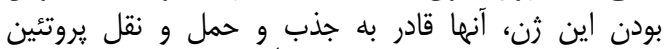

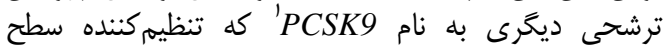

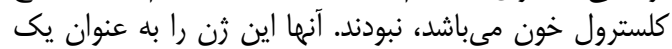
يتانسيل درمانى بالقوه براى كاهش سطح كلسترول خون و و

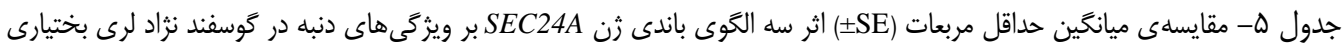
Table 5. Least squares means ( \pm se) for the effect of three banding patterns of SEC24A gene on fat-tail characteristics in Lori-Bakhtiari sheep

\begin{tabular}{|c|c|c|c|}
\hline C & B & A & صفتلا الكوى باندى \\
\hline $99 / \mathrm{r}^{\mathrm{a}} \pm \cdot / 9$ & $9 K^{\mu} / \Lambda^{b} \pm \cdot / \Lambda$ & $9 \backslash / \Delta^{c} * \pm \cdot / V$ & محيط بالاى دنبه (cm) \\
\hline$\Delta \mathrm{V} / \mathrm{ra}^{\mathrm{a}} \pm \cdot / \mathrm{\Lambda}$ & $\Delta F^{c} / \mathcal{G}^{b} \pm \cdot / V$ & $\Delta \Psi / T^{c} \pm \cdot / \varphi$ & محيط پايين دنبه (cm) \\
\hline$r N / \varphi^{a} \pm \cdot / \Delta$ & $T V / \Delta^{b} \pm \cdot / T^{c}$ & $r g / \tau^{c} \pm \cdot / \kappa$ & ارتفاع دنبه (cm) \\
\hline$F / q^{a} \pm \cdot / 1$ & $r / \Delta^{b} \pm \cdot / 1$ & $r / \pi^{c} \pm \cdot / 1$ & وزن دنبه (kg) \\
\hline
\end{tabular}

لاشه سرد، درصد جربى لاشه و دروصد دنبه مئشود را كزارش

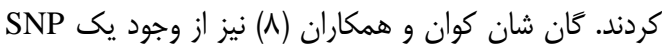

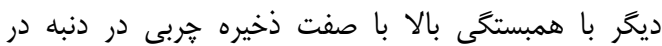

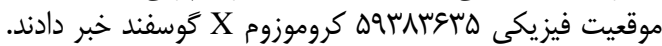

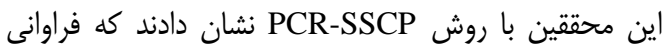

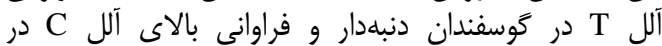

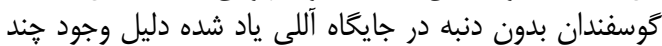
شكلى در اين جايكاه مى باشد.

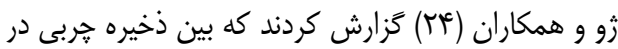

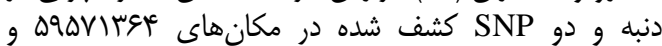
Sو X همبستخى وجود دارد. زنوتي
كوكت و همكاران (ع) گزارش كردندكه بيش از • لا ثن

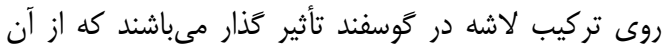

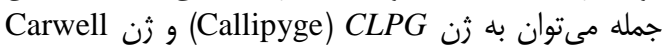

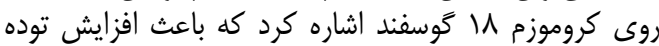

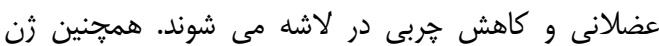
Double Muscling هييرتروفى عضلانى مىشود. اين محققين وجود

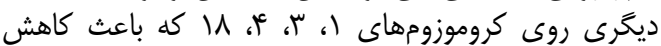

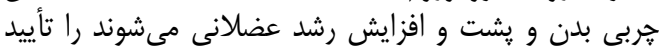

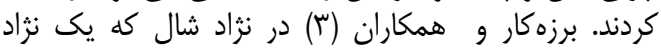

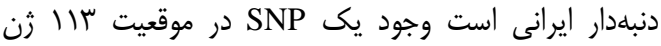

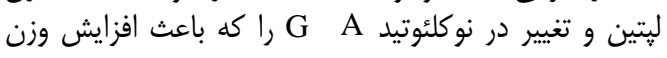

1- Proprotein convertase subtilisin/kexin type 9 
الكوى باندى C بود (شكل س). در نزراد لرى بختيارى درصد

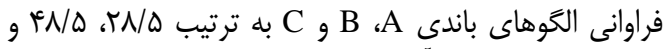

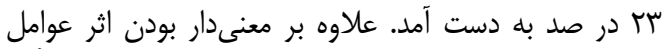

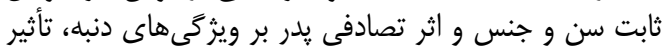

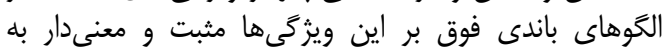

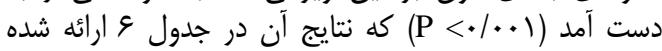
است.

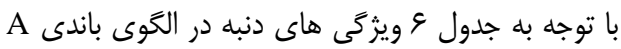

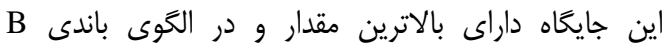

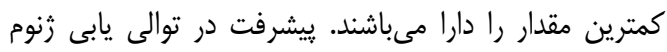

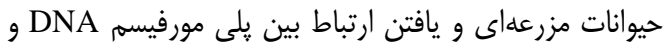

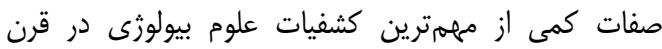

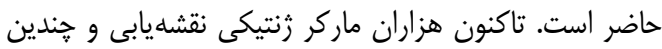
كشف شده است كه براى انتخاب مفيد واقع شدهاند.

A

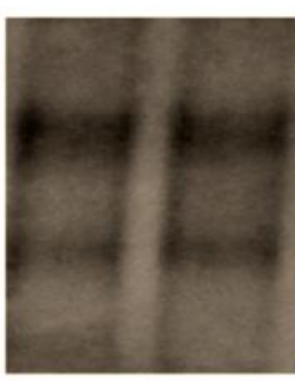

فقط الخوى C مشاهده شد) و لرى بختيارى

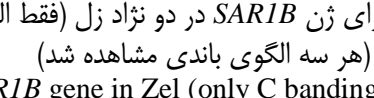
Figure 3. SSCP banding patterns for SARIB gene in Zel (only C banding pattern) and Lori-Bakhtiari (three banding
patterns) sheep breeds

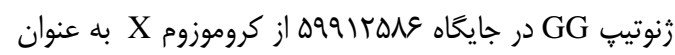
زنوتيت برتي

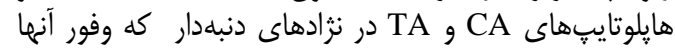

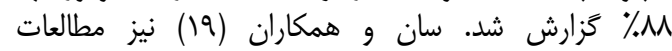

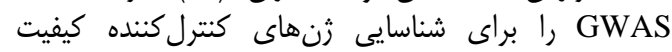

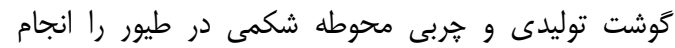

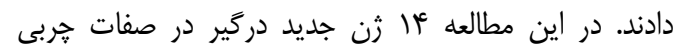

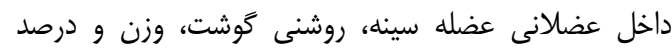
هربى محوطه شكمى شناسايى شد.

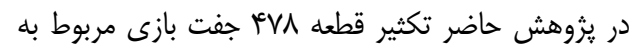

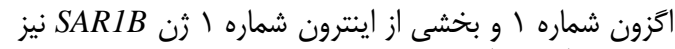

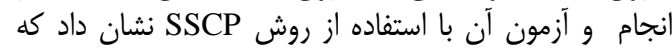

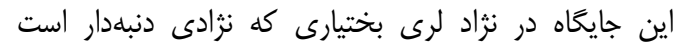

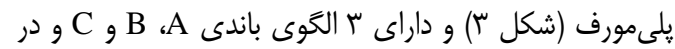
نزاد زل كه نزاد بدون دنبه است، تكى شكل و داراى
B
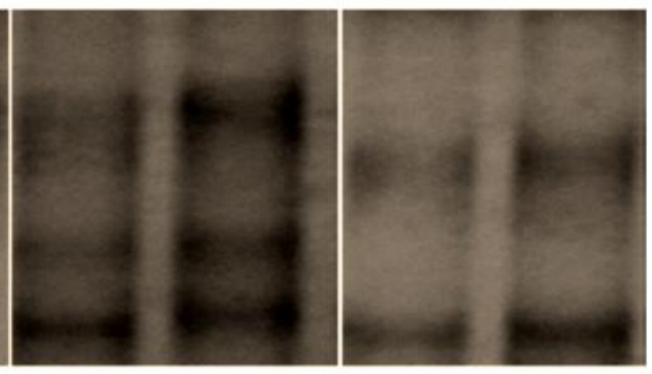

C

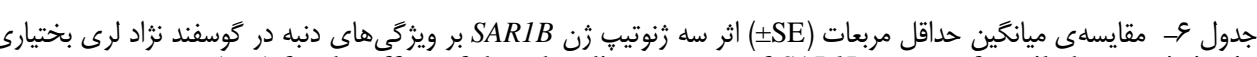
Table 6. Least square means ( \pm se) for the effect of three banding patterns of SAR1B gene on fat-tail characteristics in

\begin{tabular}{|c|c|c|c|}
\hline $\mathrm{C}$ & $\mathrm{B}$ & $\mathrm{A}$ & صفتل الكَوى باندى ٪ \\
\hline$\Delta q / \Lambda^{c} \pm \cdot / \Lambda$ & $q K / r^{D} \pm \cdot / V$ & $9 \mathrm{~V} / \Lambda^{\mathrm{a}} * \pm \cdot / \mathrm{V}$ & محيط بالاي دنبه (cm) \\
\hline$\Delta \cdot / T^{\mathrm{c}} \pm \cdot / V$ & $\Delta r / \cdot^{b} \pm \cdot / q$ & Qq/. ${ }^{\mathrm{a}} \pm \cdot / \mathrm{q}$ & محيط بايين دنبه (cm) \\
\hline$r F / \kappa^{c} \pm \cdot / \Delta$ & $r q / T^{\mathrm{b}} \pm \cdot / \kappa^{\prime}$ & $r q / r^{a} \pm \cdot / \kappa$ & ارتفاع دنبه (cm) \\
\hline$r / \Lambda^{c} \pm \cdot / 1$ & $r / r^{b} \pm \cdot / l$ & $\Delta /)^{\mathrm{a}} \pm \cdot / 1$ & وزن دنبه (kg) \\
\hline
\end{tabular}

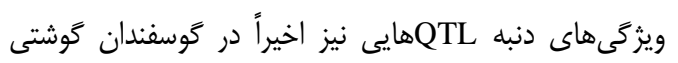

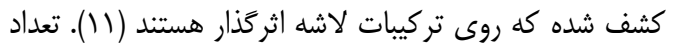

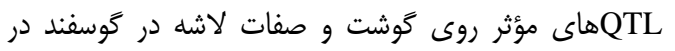

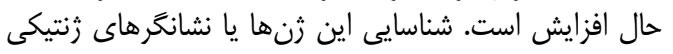

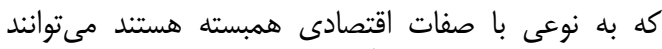

باعث افزايش رشد زنتيكى بآن صفات آن صفات شوند (9).

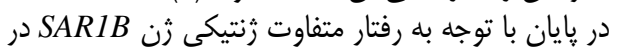

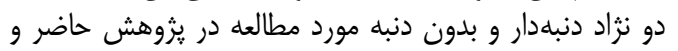

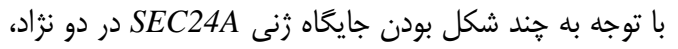

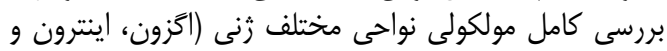

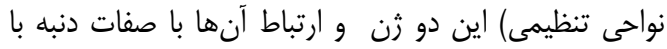
تعداد نمونههاى بيشتر ييشنهاد مئر دون

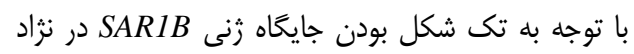

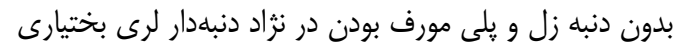

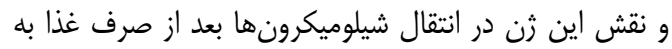

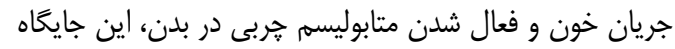

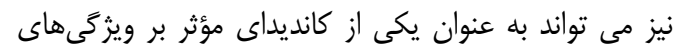

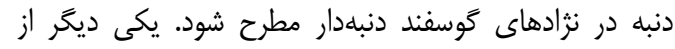

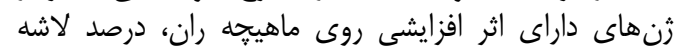

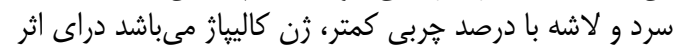

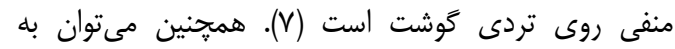
جايعاه كنترلكننده صفات كمى بـ به نام

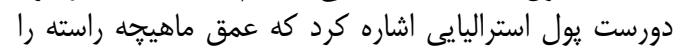

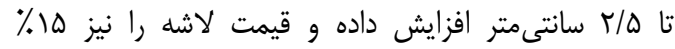

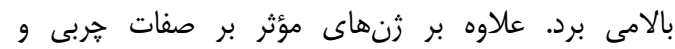


1. Archibald, A L NE Cockett, B. P Dalrymple, T. Faraut, JW Kijas, J.F Maddox, J.C. McEwan, V.Hutton Oddy, H.W. Raadsma, C. Wade, J. Wang, W. Wang and X. Xun. 2010. International Sheep Genomics Consortium: The sheep genome reference sequence: a work in progress. Animal Genetics, 41: 449-453.

2. Asadi, A., H. Moradi Shahrbabak, P. Azizi, S. Elahian and S. Abbasi. 2015. Investigation of Polymorphism in Exon 4 of GH Gene and Its Association with Growth Traits in Kermani Sheep using PCR- SSCP. Research on Animal Production, 6(12): 139-144 (In Persian).

3. Barzehkar, R., A. Salehi and F. Mahjoubi. 2009. Polymorphisms of the ovine leptingene and its association with growth and carcass traits in three Iranian sheep breeds. Iranian Journal of Biotechnology, 7: 241-246 (In Persian).

4. Bianchi, P., E. Fermo, C. Vercellati, C. Boschetti, W. Barcellini and A. Iurlo. 2009. Congenital dyserythropoietic anemia type II (CDAII) is caused by mutations in the SEC23B gene. Human Mutation, 30: 1292-1298.

5. Chen, X.W. H. Wang, K. Bajaj, P. Zhang, Z.M. Meng, D. Ma, Y. Bai, H.H. Liu, E. Adams, A. Baines, G. Yu, M.A. Sartor, B. Zhang, Z. Yi, J. Lin, S.G. Young, R. Schekman and D. Ginsburg. 2013. SEC24A deficiency lowers plasma cholesterol through reduced PCSK9 secretion. elife.elifesciences.org

6. Cockett, N.E., M.A. Smith, C.A. Bidwell, K. Segers, T.L. Hadfield, G.D. Snowder, M. Goerges and C. Charlier. 2005. The callipyge mutation and other genes that affect muscle hypertrophy in sheep. Genetic Selection Evolution, 37: S65-S81.

7. Freking, B.A., J.W. Keele, S.D. Shackelford, T.L. Wheeler, M. Koohmaraie, M.K. Nielsen and K.A. Leymaster. 1999. Evaluation of the ovine callipyge locus: III. Genotypic effects on meat quality traits. Journal of Animal Science, 77: 2336-2344.

8. GAN, S.Q., Z. Wei, S. Min, L. Huan, Y. Jing-Quan, L. Yao-Wei, G. Lei, L. Shou-Ren and W. Xin-Hua. 2013. Correlation analysis between polymorphism of the $9383635^{\text {th }}$ locus on $\mathrm{X}$ chromosome and fat-tail trait in sheep. Hereditas, 10: 1209-1218.

9. Ge, W., M.E. Davis, H.C. Hines, K.M. Irvin and R.C.M. Simmen. 2001. Association of a genetic marker with blood serum insulin-like growth factor-I concentration and growth traits in Angus cattle. Journal of Animal Science, 79: 1757-1762.

10. Gokdal, O., H. Ulker, F. Karakus, F. Cengiz, C. Temur and H. Handil. 2004. Growth, feedlot performance and carcass characteristics of Karakas and crossbred lambs (F1)(Ile de France $\mathrm{X}$ Akkaraman (G1) x Karakas) under rural farm conditions in Turkey. South African Journal of Animal Science, 34: 223-232

11. Karamichou, E., R. Richardson, G. Nute, K. Gibson and S. Bishop. 2006. Genetic analyses and quantitative trait loci detection, using a partial genome scan, for intramuscular fatty acid composition in Scottish Blackface sheep. Journal of Animal Science, 84: 3228-3238.

12. Meuwissen, T.H.E. and M.E. Goddard. 1996. The use of marker haplotypes in animal breeding schemes. Genetics Selection Evolution, 28: 161-176.

13. Moradi, M.H., A. Nejati-Javaremi, M. Moradi-Shahrbabak and J.C. McEwan. 2011. Genomic scan of selective sweeps in thin and fat tail sheen breeds for identifving of candidate regions associated with fat deposition. PhD Thesis, Tehran University, Karadj, Iran. 95 pp (In Persian).

14. Moradi, M.H., A. Nejati-Javaremi, M. Moradi-Shahrbabak, K.G. Dodds and J.C. McEwan. 2012. Genomic scan of selective sweeps in thin and fat tail sheep breeds for identifying of candidate regions associated with fat deposition. BMC Genetics, 13: 10-25.

15. Negussie, E., O. Rottmann, F. Pirchner and J. Rege. 2003. Patterns of growth and partitioning of fat depots in tropical fat-tailed Menz and Horro sheep breeds. Meat science, 64:491-498.

16. POPGENE. 1999. Version 1.32: the user friendly software for population genetic analysis. Molecular Biology and Biotechnology Centre, University of Alberta, Edmonton, AB, Canada.

17. Oligo7. 2009. Version 7. OLIGO Primer Analysis Software. Molecular Biology Insights, Inc., Cascade, CO, USA. http://www.oligo.net/

18. SAS Institute. 2000. Statistical Analysis System User's Guide. Statistics ( $8^{\text {th }}$ Edition) SAS Institute Inc. North Carolina, USA.

19. Sun, Y., G. Zhao, R. Liu, M. Zheng, Y. Hu, D. Wu, L. Zhang, P. Li and J. Wen. 2013. The identification of 14 new genes for meat quality traits in chicken using a genome-wide association study. BMC Genomics, 14: 458-464.

20. Vatankhah, M., M. Moradi-Shahrbabak, A. Nejati-Javaremi, S.R. Miraie Ashtiani and R. Vaez Torshizy. 2006. Investigation on fat-tail dimension and its relationship with Lori-Bakhtiari sheep's tail weight. Journal of Science and Technology of Agriculture and Natural Resources, Tenth year, No. III (B), autumn (In persian).

21. Wang, Q., H. Li, N. Li, L. Leng, Y. Wang and Z. Tang. 2006. Identification of single nucleotide polymorphism of adipocyte fatty Acid-Binding protein gene and its association with fatness traits in the chicken. Poultry Science, 85: 429-434.

22. Xia, W. and S.F.A. Grant. 2013. The genetics of human obesity. Annals of New York Academy of Science, 1281: 178-190.

23. Zerehdaran, S., S. Alijani and M. Salehinasab. 2016. Detecting Major Genes for Some Economic Traits in Native Fowl of Yazd Province using Different Statistical Methods. Research on Animal Production, 7(13): 163-170 (In Persian).

24. Zhu, X., N. Niu, Y. Liu, T. Du, D. Chen and X. Wang. 2006. Improvement of the sensitivity and resolution of PCR-SSCP analysis with optimized primer concentrations in PCR products. Journal of genetics, 85: 233-243. 


\title{
Study of Candidate Genes Affecting Fat-Tail Traits in Specific Region of Chromosome 5 in Lori-Bakhtiari and Zel Sheep by PCR-SSCP
}

\author{
Mazaher Safdarian', Hasan Hafezian ${ }^{2}$, Ghodrat Rahimi Mianji ${ }^{3}$ and Ayob Farhadi ${ }^{4}$ \\ 1- Graduated M.Sc. Student Department of Animal Science, Sari Agricultural Sciences and Natural Resources \\ University (Corresponding author: safdarian2014@gmail.com) \\ 2, 3 and 4- Associate Professor, Professor and Assistant Professor Department of Animal Science, Sari Agricultural \\ Sciences and Natural Resources University

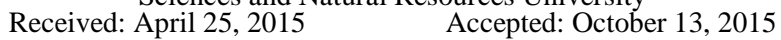

\begin{abstract}
Meat quality and carcass composition are the most important economic traits in sheep. Body fat and fat-tail are key factors affecting carcass quality and meat production in each breed, and mutations in sequences of the genes that control these traits can affect the animal performance and therefore the breeding value. The aim of this study was to investigate the polymorphism in some regions of $S A R I B, S E C 24 A$ and $V D A C 1$ genes in candidate genomic regions for fat traits on ovine chromosome 5. Blood samples were collected randomly from 300 Lori-Bakhtiari and 100 Mazandaran sheep and DNA was extracted using modified salting out method. Polymerase chain reaction (PCR) was performed for amplification of 478, 579 and $348 \mathrm{bp}$ fragments of studied genes using specific primer pairs and genotyping of samples was done by SSCP technique. The obtained results showed three banding patterns of $\mathrm{A}, \mathrm{B}$ and $\mathrm{C}$ for $S A R 1 B$ marker site in Lori-Bakhtiari sheep but it was monomorph in Zel sheep. For SEC24 gene, three banding patterns of $\mathrm{A}, \mathrm{B}$ and $\mathrm{C}$ were observed in both sheep breeds, but VDAC1 was monomorph in both sheep breed. The effect of banding patterns of $S A R 1 B$ gene on fat-tail characteristics were significant $(\mathrm{P}<0.001)$, so that the A banding pattern showed highest measurement of the upper and lower, height and weight of fat-tail. The band patterns of SEC24A gene significantly associated with fat-tail characteristics, and banding pattern of B showed highest values. Considering the monomorphic pattern of $S A R I B$ gene in Zel sheep and polymorphic pattern in fat-tailed Lori-Bakhtiary sheep, and also polyomorphic patterns of SEC24A gene in both breeds, these two marker site could be considered as potential candidate genes affecting fat tail characteristic in fat-tailed sheep breeds. More studies on different parts of these genes (exon, intron, regulatory regions) and their association with fat-tail traits is recommended for confirmation if the results.
\end{abstract}

Keywords: Fat-tail traits, SAR1B, SEC24A, Sheep, VDAC1 